\title{
Correction to: The antidepressant effect of musk in an animal model of depression: a histopathological study
}

\author{
Nasra Naeim Ayuob ${ }^{1,2} \cdot$ Soad Shaker Ali ${ }^{1}$ Mansour Suliaman ${ }^{3}$. \\ Manal Galal Abd El Wahab ${ }^{4,5} \cdot$ Samra Mansour Ahmed ${ }^{6}$
}

Published online: 23 November 2017

(C) Springer-Verlag GmbH Germany, part of Springer Nature 2017

Correction to: Cell Tissue Res (2016) 366:271-284

https://doi.org/10.1007/s00441-016-2468-9

The original publication of this paper contains mistake. Below you will find the needed corrections:

The online version of the original article can be found at https://doi.org/ 10.1007/s00441-016-2468-9

Nasra Naeim Ayuob

nasraayuob@gmail.com; nayuob@kau.edu.sa

1 Department of Anatomy, Faculty of Medicine, King Abdulaziz University, Jeddah, Saudi Arabia

2 Histology Department, Faculty of Medicine, Mansoura University, Mansoura, Egypt

3 Pharmacology Department, Faculty of Medicine, King Abdulaziz University, Jeddah, Saudi Arabia

4 Anatomy Department, Faculty of Medicine for girls, Al-Azhar University, Cairo, Egypt

5 Faculty of Nurses, National Gard, King Saud University, Jeddah, Saudi Arabia

6 Medicine Department, Faculty of Medicine, King Abdulaziz University, Jeddah, Saudi Arabia 


\section{List of corrections}

Site

Page: 275

Colum: left

Paragraph:2nd

Line: 1 st in the paragraph

Page: 279

Colum:

Paragraph: fig. 6 legend

Line:

Page: 280

Colum:

Paragraph: fig. 7 legend

Line:

Page: 281

Colum:

Paragraph: fig. 8 legend

Line:
Correction needed

CUMS significantly decreased $(p<0.001)$ the serum testosterone level...

Fig. 6 Quantitative assessment of immunoexpression of GFAP (a), caspase-3 (b), Ki67 (c), BDNF (d) and GR (e) in the hippocampus of the studied groups. Data of GFAP, caspase-3,

Ki67 expression are expressed as mean \pm SD and that of BDNF and GR is expressed as mean percent of control value $\pm \mathrm{SD}$. \#p significance versus control, *p significance versus CUMS. CUMS chronic unpredictable mild stress; FLU fluoxetine;

M musk. (ANOVA followed by a Bonferroni post hoc test)

Fig. 7 BDNF mRNA (a) and GRm RNA (c) expression levels in the hippocampus of the studied groups assessed by quantitative RT-PCR. Data are expressed as mean $\pm \mathrm{SD}(n=10)$. BDNF protein (b) and GR protein (d) expression levels in the hippocampus assessed by ELISA and expressed as percent of control value \pm SD. \#p significance versus control, *p significance versus CUMS. CUMS chronic unpredictable

mild stress; FLU fluoxetine; M musk (ANOVA followed by a Bonferroni post hoc test)

Fig. 8 Immunoexpression of GFAP (a-d), caspase-3 (e-h), Ki67 (i-l), BDNF (m-p) and GR $(\mathrm{q}-\mathrm{t})$ in the dentate gyrus of the hippocampus of the studied groups $(\mathrm{a}-1 \times 600, \mathrm{~m}-\mathrm{t} \times 400$, insets $\times 1000$ ). CUMS chronic unpredictable mild stress; FlU fluoxetine; M musk 